\title{
A CANDIDATE PLANETARY-MASS OBJECT WITH A PHOTOEVAPORATING DISK IN ORION
}

\author{
Min Fang ${ }^{1}$, Jinyoung Serena Kim ${ }^{1}$, Ilaria Pascucci ${ }^{2}$, Dániel Apai ${ }^{1,2}$, and Carlo Felice Manara ${ }^{3}$ \\ ${ }^{1}$ Department of Astronomy, University of Arizona, 933 North Cherry Avenue, Tucson, AZ 85721, USA \\ 2 Department of Planetary Sciences, University of Arizona, 1629 East University Boulevard, Tucson, AZ 85721, USA \\ ${ }^{3}$ Scientific Support Office, Directorate of Science, European Space Research and Technology Centre (ESA/ESTEC), \\ Keplerlaan 1, 2201 AZ Noordwijk, The Netherlands \\ Received 2016 September 27; revised 2016 November 16; accepted 2016 November 28; published 2016 December 12
}

\begin{abstract}
In this work, we report the discovery of a candidate planetary-mass object with a photoevaporating protoplanetary disk, Proplyd 133-353, which is near the massive star $\theta^{1}$ Ori $\mathrm{C}$ at the center of the Orion Nebula Cluster (ONC). The object was known to have extended emission pointing away from $\theta^{1}$ Ori $\mathrm{C}$, indicating ongoing external photoevaporation. Our near-infrared spectroscopic data and the location on the $\mathrm{H}-\mathrm{R}$ diagram suggest that the central source of Proplyd 133-353 is substellar ( M9.5) and has a mass probably less than 13 Jupiter mass and an age younger than 0.5 Myr. Proplyd 133-353 shows a similar ratio of X-ray luminosity to stellar luminosity to other young stars in the ONC with a similar stellar luminosity and has a similar proper motion to the mean one of confirmed ONC members. We propose that Proplyd 133-353 formed in a very low-mass dusty cloud or an evaporating gas globule near $\theta^{1}$ Ori $\mathrm{C}$ as a second generation of star formation, which can explain both its young age and the presence of its disk.
\end{abstract}

Key words: brown dwarfs - circumstellar matter - stars: pre-main sequence

\section{INTRODUCTION}

Protoplanetary disks in massive clusters can be rapidly dissipated (Balog et al. 2007; Fang et al. 2012; Guarcello et al. 2016). The main physical mechanism driving disk dispersal in these harsh environments is thought to be photoevaporation driven by UV photons from the massive stars in the clusters (Johnstone et al. 1998; Störzer \& Hollenbach 1999; Richling \& Yorke 2000; Clarke 2007; Anderson et al. 2013; Facchini et al. 2016). UV photons from massive stars ionize and heat the gas in the disk surface and induce a gas flow away from the disk when the sound speed of the gas exceeds the escape velocity (Johnstone et al. 1998; Hollenbach et al. 2000, p. 401). Direct evidence for this mechanism is provided by the large numbers of proplyds found near massive stars (O'Dell et al. 1993; Fang et al. 2012; Wright et al. 2012; Kim et al. 2016). The proplyds have cometary structures with tails pointing away from the nearby massive stars and are interpreted as the outer regions of disks of young stars that are being photoevaporated by extreme ultraviolet and far-ultraviolet (FUV) radiation from the massive stars (O'Dell et al. 1993).

Until now, the largest sample of proplyds has been found to be around $\theta^{1}$ Ori $\mathrm{C}$, which is an O6-type massive star in the Orion Nebula cluster (ONC) at a distance of $414 \mathrm{pc}$ (Menten et al. 2007). A complete sample of such proplyds has been cataloged in Ricci et al. (2008). For our investigation, with the MMT and Magellan infrared spectrograph (MMIRS; McLeod et al. 2012) mounted on the MMT telescope, we performed near-infrared spectroscopic observations of the central stars in a subsample of the proplyds (M. Fang et al. 2016 , in preparation).

In this work, we present a study of an extremely interesting case, Proplyd 133-353 in Ricci et al. (2008). Based on our spectroscopy, the central object of the proplyd has a very late spectral type ( M9.5), which suggests that it has a mass in the planetary range at the age of the ONC (1 Myr; Hillenbrand 1997). We have organized this Letter as follows: in
Section 2, we briefly introduce Proplyd 133-353; in Section 3, we describe our observations, data reduction, and delineate our data analysis; in Section 4, we present our results and discussion; followed by a summary in Section 5 .

\section{PROPLYD 133-353}

Proplyd 133-353 at R.A.(J2000.0) $=05^{\mathrm{h}} 35^{\mathrm{m}} 13.306$, decl. $(\mathrm{J} 2000.0)=-05^{\circ} 23^{\prime} 52^{\prime \prime}$.99 is located southwest of $\theta^{1}$ Ori C. The projected distance between them is about $0.11 \mathrm{pc}$. In Figure 1, we show the HST ACS color-composite image of Proplyd 133-353 using the HST images from Robberto et al. (2013). The tail can be clearly seen in the HST image in the F658N band (green emission) and extends out to $190 \mathrm{au}$. We visually inspected the $H S T$ images of the object in different bands from Robberto et al. (2013) and found that the tail can also be clearly detected in the F435W band for ACS/WFC, and the F336W and F656N bands for WFPC2, which could be due to the tail showing strong Balmer emission lines, e.g., $\mathrm{H} \alpha$ $(\mathrm{F} 658 \mathrm{~N}), \mathrm{H} \gamma(\mathrm{F} 435 \mathrm{~W})$, and Balmer continuum emission (F336W).

\section{DATA AND ANALYSIS}

\subsection{Near-infrared Spectroscopic Data}

We observed the central object of Proplyd 133-353 during the night of 2015 December 31 in the MOS mode with MMIRS, as part of our spectroscopic survey of young stars in Orion. We used the HK grism and the HK3 filter and created a mask with $0.15 \times 7^{\prime \prime}$ slits, following the MMT mask preparation procedure. This setup yields spectra from 1.25 to $2.34 \mu \mathrm{m}$, with an average spectral resolution of $\lambda / \Delta \lambda \approx 1100$. We took 8 exposures $(8 \times 300 \mathrm{~s})$ for 4 spatially dithering pairs. After the scientific exposures, we observed one telluric standard HD 34481 at a similar airmass (airmass differences $<0.02$ ) to our scientific targets.

We reduced the data using the CFA MMIRS pipeline from Chilingarian et al. (2015). The pipeline can reduce the MMIRS 


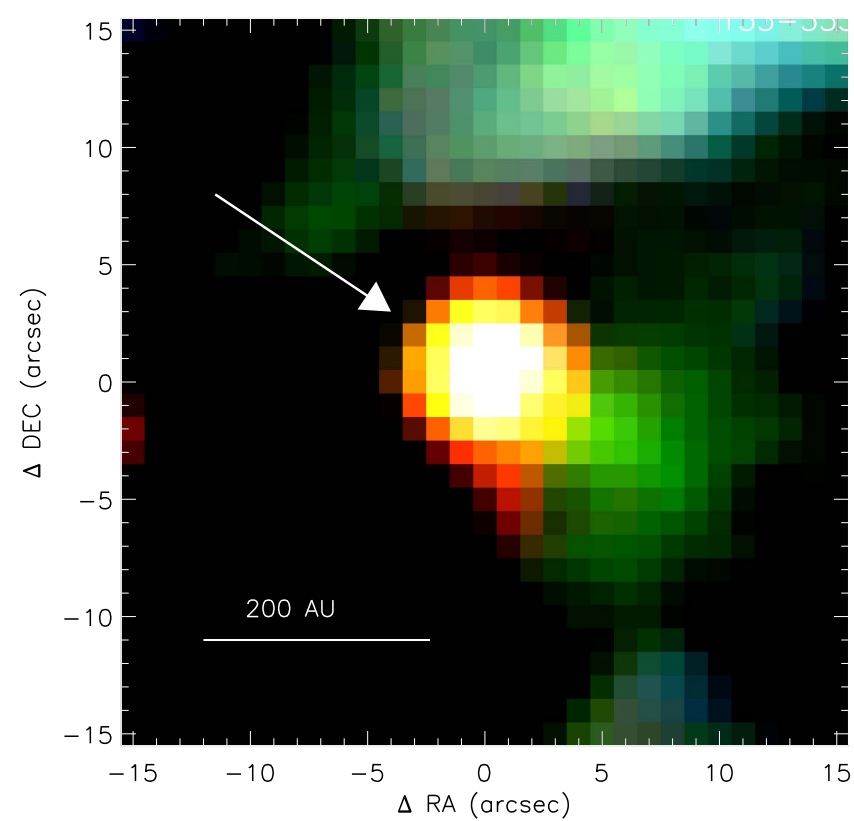

Figure 1. HST ACS color-composite image of Proplyd 133-353 (blue: F555W, green: F658N, red: F850LP). The tail of Proplyd 133-353 is obvious in the HST image in the $\mathrm{F} 658 \mathrm{~N}$ band. The arrow shows the direction from $\theta^{1}$ Ori $\mathrm{C}$ to Proplyd 133-353.

spectroscopic data in a fully automatic way. It subtracts pairs of dithered spectral exposures, performs flat-field and residual sky corrections, and rectifies the 2D spectra. The spectra are wavelength calibrated using airglow $\mathrm{OH}$ lines and corrected for the telluric absorption using telluric standard stars we observed. A detailed description of the pipeline can be found in Chilingarian et al. (2015).

\subsection{Spectral Classification}

We constructed a set of spectral templates with spectral types ranging from G5 to M9.5, using the spectra of diskless young stars collected with the instrument X-Shooter mounted on the Very Large Telescope (Manara et al. 2013, 2016, in preparation). Taking the visual extinction as a free parameter, we fit the observed spectra using the spectral templates that are reddened with the extinction law from Cardelli et al. (1989), adopting a total to selective extinction value $R_{\mathrm{V}}=3.1$. The best fit is determined by minimizing $\chi_{\text {red }}^{2}=\frac{1}{N-1} \sum_{i=1}^{N} \frac{\left(F_{\lambda, \text { obs }}-F_{\lambda, \text { template }}\right)^{2}}{\sigma_{\lambda, \text { obs }}^{2}}$, where $N$ are the numbers of the wavelength bins, $F_{\lambda \text {,obs }}$ and $F_{\lambda \text {,template }}$ are the observed fluxes and the ones from the spectral templates, respectively, within the wavelength $\lambda$ bin, and $\sigma_{\lambda, \text { obs }}$ are the errors in the observed fluxes. In Figure 2, we show a comparison of the observed spectrum of Proplyd 133-353 and the X-Shooter templates reddened with the best-fit visual extinction corresponding to the individual templates. We note that the template with spectral type M9.5 gives the best fit to the observation. However, a conversion of the spectral type M9.5 to the effective temperature $\left(T_{\text {eff }}\right)$ is still uncertain and could range from less than $2400 \mathrm{~K}$ to $\sim 2500 \mathrm{~K}$ using the different temperature scales in the literature (Luhman et al. 2003; Rajpurohit et al. 2013; Herczeg \& Hillenbrand 2014). To avoid this uncertainty, we fit the spectrum of Proplyd 133-353 directly with the BT-Settl atmospheric models with solar abundances from Asplund et al.
(2009) and a surface gravity $\log g=3.5$, which is suitable for the very low-mass young substellar objects (Baraffe et al. 2015). As with our procedure for the X-Shooter templates, we took the visual extinction as a free parameter and searched for the best-fit atmospheric models. Figure 2 shows a comparison of the observed spectrum and the reddened BT-Settl models. We found the best-fit BT-Settl models are the ones with the effective temperatures 2400 and $2500 \mathrm{~K}$ with the best-fit visual extinction of 0.3 and $0.7 \mathrm{mag}$, respectively. Thus, in this work, we adopt $T_{\text {eff }}=2450 \pm 50 \mathrm{~K}$ and $A_{\mathrm{V}}=0.5 \pm 0.2$ for the central object of Proplyd 133-353.

\section{RESULTS AND DISCUSSION}

\subsection{Masses and Ages}

We obtained the near-infrared photometry of Proplyd 133353 from Robberto et al. (2010). The stellar luminosity of Proplyd 133-353 was derived using dereddened $J$-band photometry, and the $J$-band bolometric correction $\left(B C_{J}=2.12\right)$ for $T_{\text {eff }}=2450 \pm 50 \mathrm{~K}$ calculated with the BT-Settl models, assuming a distance of $414 \mathrm{pc}$ (Menten et al. 2007). The stellar luminosity $\left(L_{\star}\right)$ of Proplyd 133-353 is estimated to be $0.021 \pm 0.004 L_{\odot}$. The uncertainty of $L_{\star}$ is calculated considering the uncertainty in the $J$-band magnitude, the $T_{\text {eff }}$ value, and the extinction. In Figure 3 (left), we placed Proplyd 133-353 in the H-R diagram. As a comparison, we also show other young stars in the ONC with data collected from Da Rio et al. (2012), as well as the pre-main-sequence (PMS) evolutionary tracks from Baraffe et al. (2015). In the $\mathrm{H}-\mathrm{R}$ diagram, Proplyd 133-353 is above the youngest PMS isochrone ( $\sim 0.5 \mathrm{Myr})$ from Baraffe et al. (2015) and has a much cooler $T_{\text {eff }}$ compared with other young stars, but shows an $L_{\star}$ similar to a $0.05 M_{\odot}$ brown dwarf at 1 Myr. Recently, Kounkel et al. (2016) found the mean distance of the ONC to be $388 \pm 5 \mathrm{pc}$. Using this distance estimate, the $L_{\star}$ of Proplyd 133-353 will be reduced by a factor of 1.14 , which moves the object down on the $\mathrm{H}-\mathrm{R}$ diagram very slightly. Therefore, the object is still very young (age $<0.5 \mathrm{Myr}$ ) even with this new distance estimate.

Due to the lack of PMS evolutionary tracks at ages younger than 0.5 Myr in Baraffe et al. (2015), it is not possible to derive its mass and age directly through comparison to the PMS evolutionary tracks. Here, we tentatively constrain its mass using the $0.5 \mathrm{Myr}$ isochrone from Baraffe et al. (2015). On this isochrone, the mass of an object with $T_{\text {eff }}=2450$ is $\sim 13$ Jupiter mass $\left(M_{\mathrm{J}}\right)$, which is much lower than the known lowest mass $\left(42 M_{\mathrm{J}}\right.$ ) for the objects at the centers of proplyds in ONC in the literature (Robberto et al. 2008). However, this mass might be an upper limit for Proplyd 133-353 since the $T_{\text {eff }}$ values of young low-mass substellar objects seem to decrease during their evolution. The dividing line between a brown dwarf and a planet is around $13 M_{\mathrm{J}}$, as defined by the deuterium fusion mass limit (Burrows et al. 1997; Spiegel et al. 2011). Therefore, Proplyd 133-353 could be a planetarymass object with an age younger than $0.5 \mathrm{Myr}$. We also use the PMS evolutionary tracks from D'Antona \& Mazzitelli (1997), which give a mass $\sim 25 M_{\mathrm{J}}$ and an age $\sim 4000$ year. We note that evolutionary models at these young ages and very low masses have significant uncertainties (Close et al. 2005; Konopacky et al. 2010); therefore, in this work, we do not attempt to conclusively determine the nature of the object. 


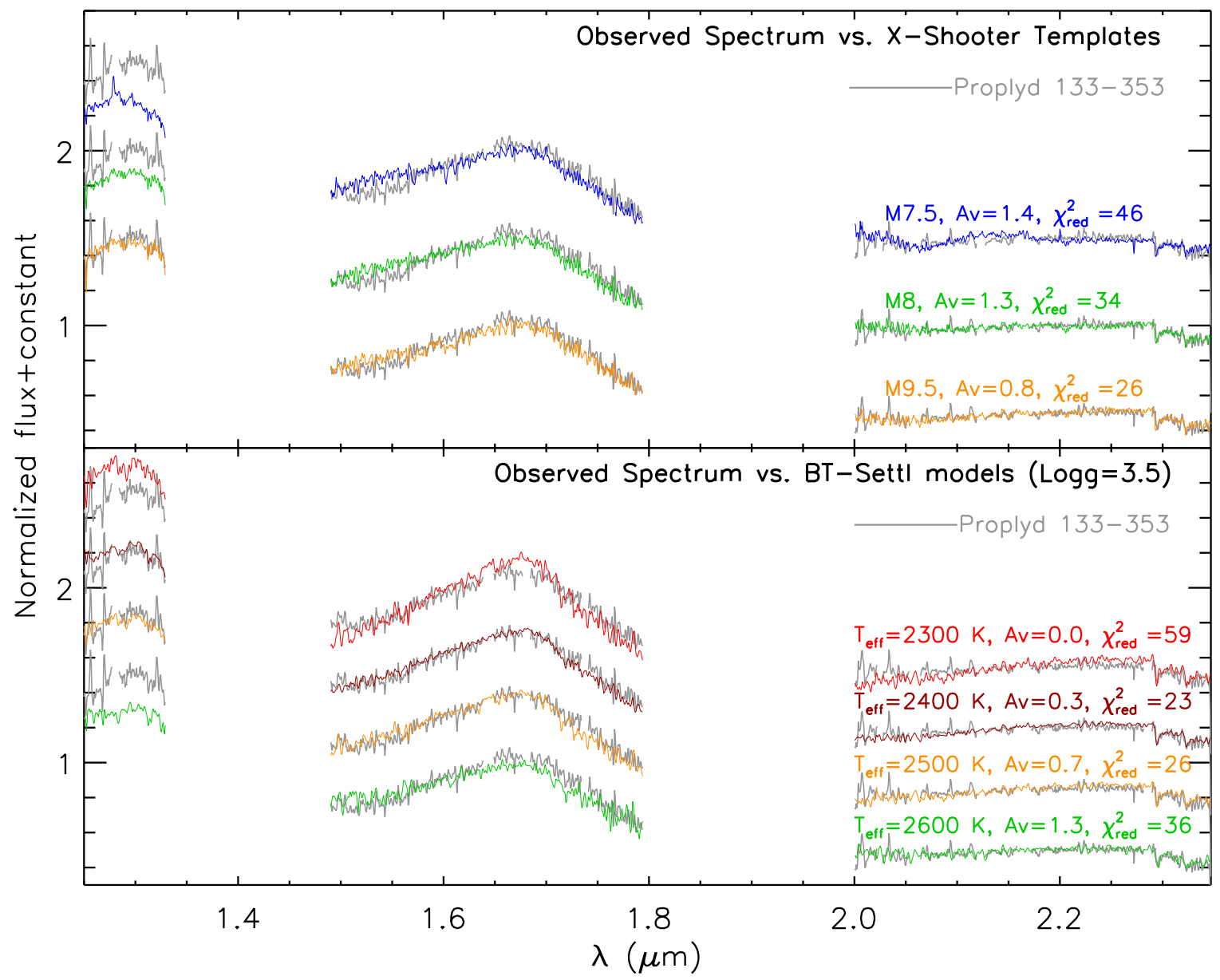

Figure 2. MMIRS spectrum of Proplyd 133-353 (gray lines). The reddened X-Shooter templates (top) and the BT-Settl atmospheric models (bottom) are shown for comparison. For individual templates or models, their spectral types or effective temperature, as well as the best-fit visual extinctions and the $\chi_{\text {red }}^{2}$ are marked.

Whether in the brown dwarf or planetary-mass category, Proplyd 133-353 is an interesting object for a detailed study.

\subsection{Is Proplyd 133-353 a Member of the ONC?}

As described in Section 2, Proplyd 133-353 has a tail pointing away from $\theta^{1}$ Ori $\mathrm{C}$, which indicates that its disk is being dissipated by the massive star. Thus, Proplyd 133-353 should be a member of the ONC. Here, we will discuss further evidence for the ONC membership of Proplyd 133-353 based on its X-ray properties and proper motion.

Proplyd 133-353 has been detected by the Chandra space telescope in Chandra Orion Ultradeep Project (Getman et al. 2005). In Figure 3 (right), we show its X-ray luminosity $\left(L_{\mathrm{X}}\right)$ over the $0.5-8.0 \mathrm{keV}$ band versus its stellar luminosity and compare it with the values from other young stars in the ONC with the $L_{\mathrm{X}}$ from Getman et al. (2005) and $L_{\star}$ from Da Rio et al. (2012). The $L_{X} / L_{\star}$ ratio of Proplyd 133-353 is similar to those of other young stars with similar stellar luminosity.

According to the PMS evolutionary models (Baraffe et al. 2015), the expected $L_{\star}$ for Proplyd 133-353 at an age of $1 \mathrm{Myr}$ is $0.00307 L_{\odot}$, corresponding to its $T_{\text {eff. }}$ If Proplyd 133-353 is a foreground $1 \mathrm{Myr}$ old planet, the expected distance could be $\sim 160 \mathrm{pc}$, corresponding to the intrinsic luminosity and the observed brightness. If that is the case, we would expect that Proplyd 133-353 shows a different proper motion from the ones of other young stars in the ONC.
We derive the proper motion from the $H S T$ archive images in the $\mathrm{F} 775 \mathrm{~W}$ band observed at two epochs (2005 April versus 2015 February). ${ }^{4}$ In Figure 4, we show the second-epoch HST image. The image has been calibrated in astrometry to the firstepoch image using the common ONC members. We excluded Proplyd 133-353 in the astrometric calibration; therefore, we can measure the proper motion of the proplyd relative to the ONC. As a comparison, in Figure 4, we also show the positions of the stars in the first-epoch $H S T$ image. From the image pair, we obtain a relative proper motion for Proplyd 133-353, of $\mu_{\alpha}$

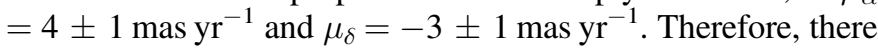
is no significant proper motion of Proplyd 133-353 relative to the ONC. However, we cannot exclude the possibility that Proplyd 133-353 and the ONC have different motions in the line of sight, given the spectral resolution $(\lambda / \Delta \lambda \approx 1100)$ of our MMIRS data.

In conclusion, the arguments presented above, especially the cometary structure with a tail pointing away from $\theta^{1}$ Ori $\mathrm{C}$ and the X-ray luminosity, strongly support that Proplyd $133-353$ is a member of the ONC.

\footnotetext{
4 The first-epoch HST image is obtained from Robberto et al. (2013), and the second-epoch HST image (PropID 13826) is downloaded from http://hla.stsci. edu/.
} 

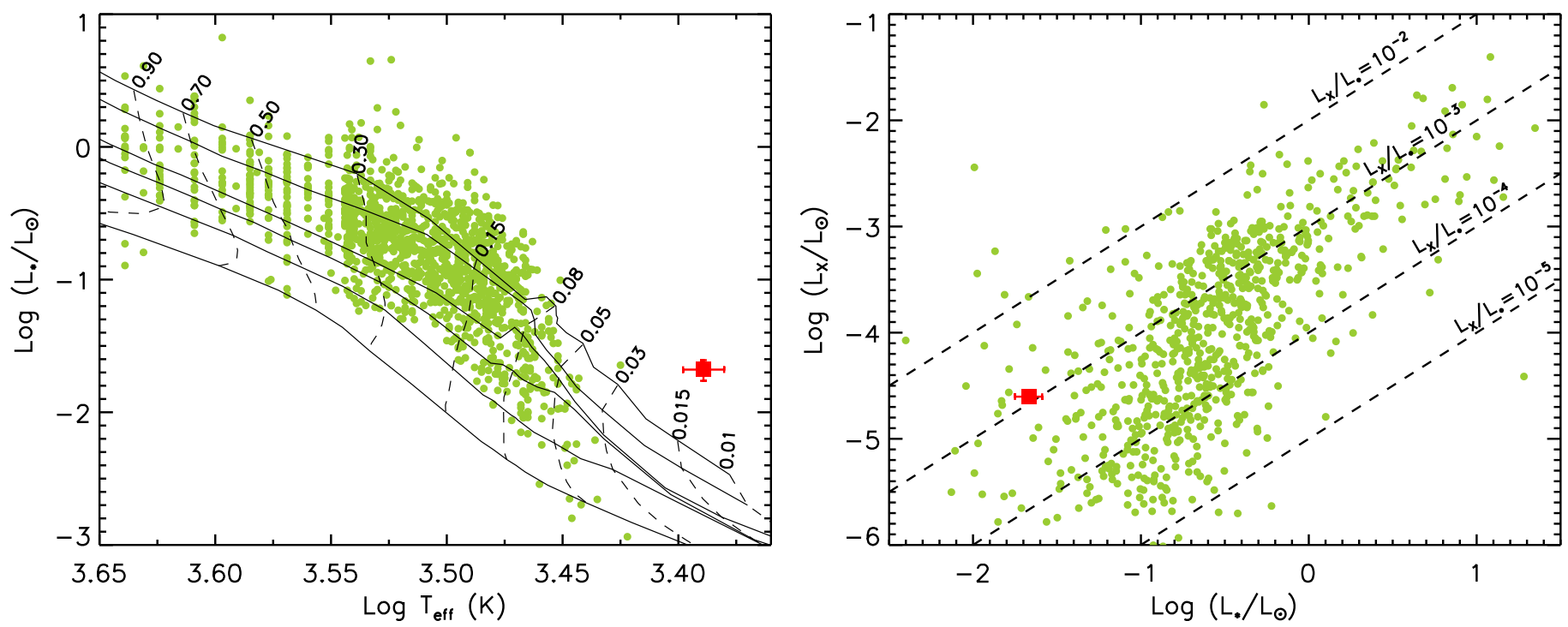

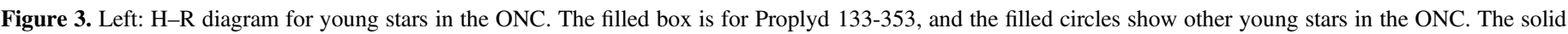

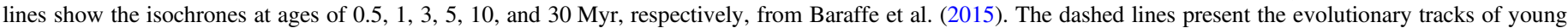

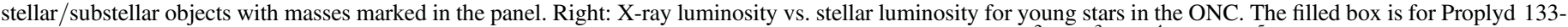
353 , and the filled circles show other young stars in the ONC. The dashed lines mark $L_{\mathrm{X}} / L_{*}$ ratios of $10^{-2}, 10^{-3}, 10^{-4}$, and $10^{-5}$.

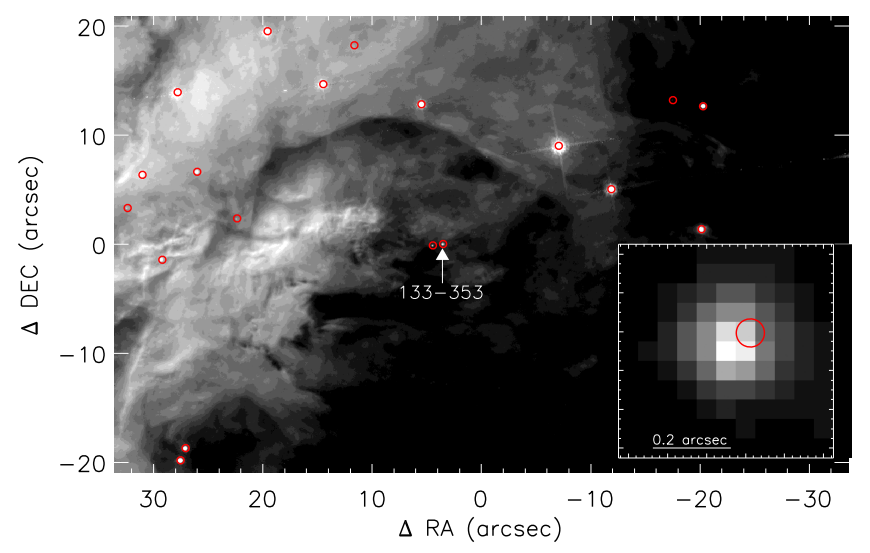

Figure 4. HST F775W-band image of the field around Proplyd 133-353 observed in 2015 February. The arrow marks the position of Proplyd 133-353. The open circles show the positions of the stars on the HST F775W-band image observed in 2005 April. The inset zooms in on Proplyd 133-353.

\subsection{The Possible Nature of Proplyd 133-353}

In the following, we will compare the different scenarios for the origin and nature of Proplyd 133-353.

Theoretical calculations predict that mass-loss rates from disks due to photoevaporation can be on the order of $10^{-7} M_{\odot} \mathrm{yr}^{-1}$ within a distance of $0.2 \mathrm{pc}$ from the ionizing massive star $\theta^{1}$ Ori C (Störzer \& Hollenbach 1999), which is confirmed by spectroscopic observations (Henney \& O'Dell 1999). The photoevaporation process can effectively dissipate protoplanetary disks outside the gravitation radii $\left(r_{\mathrm{g}}\right)$, where the escape velocities equal the speed of sound, which is in turn determined by the UV heating (Hollenbach et al. 2000, p. 401). With such high mass-loss rates, we would expect a very short disk lifetime for Proplyd 133-353, which is $\sim 10^{3}$ years with an assumption of a typical ratio $(\sim 100)$ between stellar mass and disk mass.

It is well known that there are many tiny dusty clouds, called "globulettes," around H II regions (De Marco et al. 2006; Gahm et al. 2007; Grenman \& Gahm 2014). The mean mass of the globulettes is around several $\times 10 M_{\mathrm{J}}$, and their mean radius is $\sim 4000$ au (Gahm et al. 2007). These globulettes are undergoing photoevaporation by UV photons from the massive stars in $\mathrm{H}$ II regions. However, the shocks generated by photoionization, as well as the surrounding warm gas, can also exert external pressure on the globulettes and drive their collapse to form brown dwarfs and free-floating planets before they are eroded by photoevaporation (Gahm et al. 2007). Such a second-generation formation of brown dwarfs and free-floating planets has been confirmed by the discovery of dense cores in some of the globulettes (Gahm et al. 2013). Haworth et al. (2015) doubt the possibility that the external pressure in the form of radiation or ram pressure can drive globulettes to form brown dwarfs or free-floating planets, and instead they propose the collapse of the globulettes might be triggered by their collisions with the shells that border the expanding $\mathrm{H}$ II regions. If Proplyd 133-353 was formed in such a globulette near $\theta^{1}$ Ori $\mathrm{C}$ due to either mechanism, we might explain both its younger age (with respect to the one of the ONC) and the lifetime of its disk since its parental cloud can protect it from direct photoevaporation for a timescale of $10^{5}-10^{6}$ years (Gahm et al. 2007; Haworth et al. 2015).

Finally, we explore an alternative scenario for the formation of Proplyd 133-353: instead of a photoevaporating disk it may be an evaporating gaseous globule (EGG). In the vicinity of massive stars, a prestellar core can be eroded by the ionizing radiation from massive stars and may form a free-floating brown dwarf or planetary-mass object (Hester et al. 1996; Whitworth \& Zinnecker 2004). The final mass of the formed object can be approximated as $0.010 M_{\odot}\left(\frac{a_{1}}{0.3 \mathrm{~km} \mathrm{~s}^{-1}}\right)^{6}\left(\frac{\dot{N}_{\mathrm{Lyc}}}{10^{50} \mathrm{~s}^{-1}}\right)^{-1 / 3}\left(\frac{n_{0}}{10^{3} \mathrm{~cm}^{-3}}\right)^{-1 / 3}$ (Whitworth \& Zinnecker 2004), where $a_{1}$ is the isothermal sound speed in the neutral gas of the core, $\dot{N}_{\mathrm{Lyc}}$ is the emission rate of Lyman continuum photons, and $n_{0}$ is the number density of protons in the $\mathrm{HII}$ region around the core. In ONC, we take $\dot{N}_{\text {Lyc }} \sim 2.6 \times 10^{49} \mathrm{~s}^{-1}$ (Bertoldi \& Draine 1996), and $n_{0} \sim 5.5 \times 10^{3}$ around Proplyd 133-353 (Weilbacher et al. 2015). Assuming $a_{1} \sim 0.23-0.36 \mathrm{~km} \mathrm{~s}^{-1}$ for a prestellar 
core with a gas temperature of $15-35 \mathrm{~K}$, the expected final mass of the object formed in the core near Proplyd 133-353 would be around $2-28 M_{\mathrm{J}}$, which is consistent with the mass $\left(13 M_{\mathrm{J}}\right)$ of Proplyd 133-353.

\section{CONCLUSION}

We have presented new near-infrared spectroscopy of Proplyd 133-353, a candidate photoevaporating disk given its cometary shape. The near-infrared spectroscopic data and and the location on the $\mathrm{H}-\mathrm{R}$ diagram suggests that its central source is an M9.5-type substellar object with a mass $\left(\lesssim 13 M_{\mathrm{J}}\right)$ close to the planetary regime and an age younger than $0.5 \mathrm{Myr}$. Proplyd 133-353 has been detected by Chandra and presents a similar ratio of $\mathrm{X}$-ray luminosity to stellar luminosity as other young stars in the ONC with similar stellar luminosity. The comparison of two-epoch HST images suggests that Proplyd 133-353 has a similar proper motion to the one of the ONC. We explore two possibilities to explain the existence of Proplyd 133-353 near $\theta^{1}$ Ori $C$ and propose that Proplyd 133353 was formed in a very low-mass dusty cloud or an EGG near $\theta^{1}$ Ori $\mathrm{C}$ in a second generation of star formation, which can explain both its young age and the estimated lifetime of its disk.

The discovery of Proplyd 133-353 may provide a clue to understanding the significant peak at $10-20 M_{\mathrm{J}}$ of the ONC initial mass function (Muench et al. 2002; Drass et al. 2016). The second-generation star formation in the very low-mass globulettes or in the EGGs could overpopulate the objects of the ONC within such a mass range.

Many thanks to Dr. Carmen Ortiz Henley for helping improve the language of the manuscript and the anonymous referee for comments that help to improve this Letter. This material is based upon work supported by the National Aeronautics and Space Administration under Agreement No. NNX15AD94G for the program "Earths in Other Solar Systems." The results reported herein benefited from collaborations and/or information exchange within NASAs Nexus for Exoplanet System Science (NExSS) research coordination network sponsored by NASA's Science Mission Directorate. CFM gratefully acknowledges an ESA Research Fellowship. This research is based on observations made with the NASA/ ESA Hubble Space Telescope, and obtained from the Hubble Legacy Archive, which is a collaboration between the Space Telescope Science Institute (STScI/NASA), the Space Telescope European Coordinating Facility (ST-ECF/ESA), and the Canadian Astronomy Data Centre (CADC/NRC/CSA).

\section{REFERENCES}

Anderson, K. R., Adams, F. C., \& Calvet, N. 2013, ApJ, 774, 9

Asplund, M., Grevesse, N., Sauval, A. J., \& Scott, P. 2009, ARA\&A, 47, 481 Balog, Z., Muzerolle, J., Rieke, G. H., et al. 2007, ApJ, 660, 1532 Baraffe, I., Homeier, D., Allard, F., \& Chabrier, G. 2015, A\&A, 577, A42 Bertoldi, F., \& Draine, B. T. 1996, ApJ, 458, 222

Burrows, A., Marley, M., Hubbard, W. B., et al. 1997, ApJ, 491, 856

Cardelli, J. A., Clayton, G. C., \& Mathis, J. S. 1989, ApJ, 345, 245

Chilingarian, I., Beletsky, Y., Moran, S., et al. 2015, PASP, 127, 406

Clarke, C. J. 2007, MNRAS, 376, 1350

Close, L. M., Lenzen, R., Guirado, J. C., et al. 2005, Natur, 433, 286

D’Antona, F., \& Mazzitelli, I. 1997, MmSAI, 68, 807

Da Rio, N., Robberto, M., Hillenbrand, L. A., Henning, T., \& Stassun, K. G. 2012, ApJ, 748, 14

De Marco, O., O’Dell, C. R., Gelfond, P., Rubin, R. H., \& Glover, S. C. O. 2006, AJ, 131, 2580

Drass, H., Haas, M., Chini, R., et al. 2016, MNRAS, 461, 1734

Facchini, S., Clarke, C. J., \& Bisbas, T. G. 2016, MNRAS, 457, 3593

Fang, M., van Boekel, R., King, R. R., et al. 2012, A\&A, 539, A119

Gahm, G. F., Grenman, T., Fredriksson, S., \& Kristen, H. 2007, AJ, 133, 1795

Gahm, G. F., Persson, C. M., Mäkelä, M. M., \& Haikala, L. K. 2013, A\&A, 555, A57

Getman, K. V., Flaccomio, E., Broos, P. S., et al. 2005, ApJS, 160, 319

Grenman, T., \& Gahm, G. F. 2014, A\&A, 565, A107

Guarcello, M. G., Drake, J. J., Wright, N. J., et al. 2016, ApJS, in press (arXiv:1605.01773)

Haworth, T. J., Facchini, S., \& Clarke, C. J. 2015, MNRAS, 446, 1098

Henney, W. J., \& O'Dell, C. R. 1999, AJ, 118, 2350

Herczeg, G. J., \& Hillenbrand, L. A. 2014, ApJ, 786, 97

Hester, J. J., Scowen, P. A., Sankrit, R., et al. 1996, AJ, 111, 2349

Hillenbrand, L. A. 1997, AJ, 113, 1733

Hollenbach, D. J., Yorke, H. W., \& Johnstone, D. 2000, in Protostars and Planets IV, ed. V. Mannings, A. P. Boss, \& S. S. Russell (Tucson, AZ: Univ. Arizona Press), 401

Johnstone, D., Hollenbach, D., \& Bally, J. 1998, ApJ, 499, 758

Kim, J. S., Clarke, C. J., Fang, M., \& Facchini, S. 2016, ApJL, 826, L15

Konopacky, Q. M., Ghez, A. M., Barman, T. S., et al. 2010, ApJ, 711, 1087

Kounkel, M., Hartmann, L., Loinard, L., et al. 2016, ApJ, in press (arXiv:1609. 04041)

Luhman, K. L., Stauffer, J. R., Muench, A. A., et al. 2003, ApJ, 593, 1093

Manara, C. F., Testi, L., Rigliaco, E., et al. 2013, A\&A, 551, A107

McLeod, B., Fabricant, D., Nystrom, G., et al. 2012, PASP, 124, 1318

Menten, K. M., Reid, M. J., Forbrich, J., \& Brunthaler, A. 2007, A\&A, 474, 515

Muench, A. A., Lada, E. A., Lada, C. J., \& Alves, J. 2002, ApJ, 573, 366

O’Dell, C. R., Wen, Z., \& Hu, X. 1993, ApJ, 410, 696

Rajpurohit, A. S., Reylé, C., Allard, F., et al. 2013, A\&A, 556, A15

Ricci, L., Robberto, M., \& Soderblom, D. R. 2008, AJ, 136, 2136

Richling, S., \& Yorke, H. W. 2000, ApJ, 539, 258

Robberto, M., Ricci, L., Da Rio, N., \& Soderblom, D. R. 2008, ApJL, 687, L83

Robberto, M., Soderblom, D. R., Bergeron, E., et al. 2013, ApJS, 207, 10

Robberto, M., Soderblom, D. R., Scandariato, G., et al. 2010, AJ, 139, 950

Spiegel, D. S., Burrows, A., \& Milsom, J. A. 2011, ApJ, 727, 57

Störzer, H., \& Hollenbach, D. 1999, ApJ, 515, 669

Weilbacher, P. M., Monreal-Ibero, A., Kollatschny, W., et al. 2015, A\&A, 582, A114

Whitworth, A. P., \& Zinnecker, H. 2004, A\&A, 427, 299

Wright, N. J., Drake, J. J., Drew, J. E., et al. 2012, ApJL, 746, L21 